\title{
DEATH RATES IN THE CALORIE MODEL
}

\author{
Martin Machay ${ }^{1}$ \\ ${ }^{1}$ Department of Economics, Faculty of Business and Economics, Mendel University in Brno, Zemědělská 1, \\ 61300 Brno, Czech Republic
}

\begin{abstract}
MACHAY MARTIN. 2016. Death Rates in the Calorie Model. Acta Universitatis Agriculturae et Silviculturae Mendelianae Brunensis, 64(6): 2053-2058.

The Calorie model unifies the Classical demand and the supply in the food market. Hence, solves the major problem of Classical stationary state. It is, hence, formalization of the Classical theory of population. The model does not reflect the imperfections of reality mentioned by Malthus himself. It is the aim of this brief paper to relax some of the strong assumptions of the Calorie model to make it more realistic. As the results show the political economists were correct. The death resulting from malnutrition can occur way sooner than the stationary state itself. Moreover, progressive and retrograde movements can be easily described by the death rate derived in the paper.

JEL Classification: J11, Q11, Q15, Q21, Y90
\end{abstract}

Keywords: stationary state, food, population, the calorie model, Malthus

\section{INTRODUCTION}

Malthusian principle was tempting for economists since it was stated for the first time in 1798 The relation between food supply and food demand stayed more or less in the area of interest ever since. The original idea about growth of population exceeding the capacity of an economy to produce the means of subsistence leads to only one logical conclusion - stationary state. A moment in time when first people begin to die from malnutrition.

The original thought was first moved from the mainstream economics due to contemporary realities when a capacity to produce food increased tremendously (Madsen et al., 2010) and then it was seemingly solved by the almighty power of substitution mentioned by Jevons. Malthusian principle became a fringe economic topic for several decades with resurfacing time to time. Later it was reformulated by Kremer (1993) in a modern fashion - the growth rates became endogenous in the model (see also Golosov et al., 2007) and means of subsistence became a mere arbitrarily set level of income. Which per se moved the interpretation from the original ideas of the Classical school. With endogenous growth rates the dismal future was eventually simplified to a utilitarian problem of parents (Becker et al., 1999) and they are enticing to be estimated by modern econometric tools (Birchenall, J. A., 2015). Kremer's model is, thus, a Neoclassical one.
Even though the modern interpretation is elegant and intuitive it is in great contradiction to Malthus thoughts. Malthus saw the growth of population as exogenous and in no way dependent on self-control of an individual: "I see no way by which man can escape from the weight of this law which pervades all animated nature." (Malthus, 1798) The same can be said about the "power in the earth to produce subsistence for man."

As a consequence the Malthusian thoughts have been reduced to a simple situation of growth of population exceeding the capacity to produce the means of subsistence and words and ideas of Malthus were reformulated or changed completely. The original Malthusian framework have been formulated quite recently in the form of the Calorie model (Machay, 2012) which builds strictly on Malthusian thoughts and within the Classical School approach using modern mathematical tools to unify the Classical/Malthusian demand for and supply of food. The Calorie model is just a mere formalization of ideas of Malthus.

The Calorie model stands on a strong assumption of equal distribution of calories. Malthus, however, did recognize such an assumption as unrealistic. Using his words: "The poor consequently must live much worse, and many of them be reduced to severe distress" (Malthus, 1798). He focused on the stationary state but he admitted that there are deaths preceding the stationary state itself. 
It is the aim of this short paper to examine the death rates within the Malthusian framework by relaxing the strong assumption of the Calorie model. The task at hand, hence, solves a Classical problem within the Classical School. It is a complement to the Classical School and in no way in the opposition to the neoclassical model as such. The paper is divided into two parts. First part introduces the Calorie model formally. The second part then examines the deaths in the stationary state and relaxes the strong assumption of the model about the equal distribution of calories within the global economy.

\section{The Calorie model}

Due to non-uniformity of the demand for food and the supply of food that do not share the same dimension in the Classical school the model assumes that what Classics intended to say was the demand for and supply of calories. Calories are energy that powers the organic bodies. Having too little of them the body shuts down. Once again we can use Malthus's own words for expressing the modern knowledge about human bodies: "By the law of our nature which makes food necessary to the life of man..." (Malthus, 1798).

It is not the goal of this section to present all details of the Calorie model. For details see the original article from 2012 (Machay).

The Calorie model places calories needed to sustain the population against the calories production capacity of the planet. Formally the calories consumption surplus $\left(c_{s}\right)$ can be constructed as

$c_{S}=\frac{A(t) a_{T}}{L(t) c_{N}}$

where $A(t)$ is technological level in time $t, a_{T}$ represents amount of calories produced naturally on all disposable land on the planet without being affected by human activity, $L(t)$ is the total population in time $t$, and $c_{N}$ stands for the minimal amount of calories one average person must consume per unit of time. Hence, it is obvious that the $c_{S}$ represents the proportion between the available amount of calories and the minimum necessary to feed the population in any given time $t$.

Discounting (1) to the beginning of time and equalizing origins to one yields

$c_{S}=\frac{e^{g t} a_{T}}{e^{n t} c_{N}}=e^{(g-n) t} \frac{a_{T}}{c_{N}}$.

As we can see the surplus is, hence, strictly dependent - among obvious ones - on the time we are in and the initial endowment of the planet. Under these circumstances the Classical stationary state occurs in time $t_{p}$

$t_{P}=\frac{\log c_{N}-\log a_{T}}{g-n}$
The Calorie model then implies that from logical reasons $g<n$ must hold. Hence, the technological growth must be smaller than the growth of population for the stationary state to occur. This is the proof of Malthusian principle.

The Calorie model is also accompanied by the calorie price that is constructed to fulfill several convenient conditions:

1) if $\Delta c_{s}$ when $c_{s}\left(t_{1}\right)$ and $c_{s}\left(t_{2}\right)$ are very large then $\Delta p \rightarrow 0$

2) $\frac{\partial a_{T}}{\partial t}<0 \Rightarrow \frac{\partial p(t)}{\partial t}>0$

3) $\frac{\partial c_{s}}{\partial t}<0 \Rightarrow \frac{\partial p(t)}{\partial t}>0$

4) $\lim _{t \rightarrow t_{p}} p(t)=\infty$.

In the times of large consumption surpluses the price fluctuates insignificantly. Natural conditions affecting the $a_{T}$ have a reverse effect to the price. The price is rising as the economy moves towards the stationary state and finally the price must be sky high when the stationary state is near. To avoid a dull and simple discounted price trajectory the $p(t)$ is arbitrarily set in the model as

$$
p(t)=\frac{\gamma}{e^{g t} a_{T}-e^{n t} c_{N}}
$$

where $\gamma$ is the $p(t)$ for the very last death-free/surplus calorie. Thus, the price of the last available free calorie before first people in the model will begin to die.

The visualization of the model is provided in the Fig. 1.

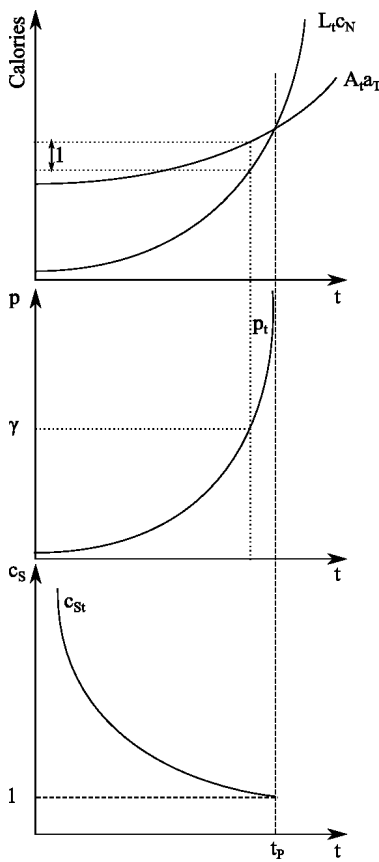

1: The Calorie model (Machay, 2012; pp. 176). 
The Calorie model as it is constructed stands on two relatively strong assumptions.

First, the food in the form of calories is distributed equally within the society and the global economy. Second, the growth rates of population and technological level are exogenous and are constant. On the other hand we can see that the price is endogenous.

The model itself leads to already mentioned theorem that the growth of population must be larger than the growth of technological level for stationary state to occur. From mentioned assumptions, specifications, and theorem it is clear why the Calorie model can be considered as a formulation of Malthusian thoughts.

The original article (Machay, 2012) develops and uses the Calorie model to explain the terraformation production as a consequence of rational economic behavior of economic agents. While the solution to stationary state offered in the article seems rather distant from the time point of view it is obvious that the deaths from malnutrition did and will take place sooner than the stationary state itself or its solution in the form of terraformation.

From this reason the Calorie model fails to explain our contemporary realities while it forecasts the solution.

Moreover, from two assumptions of the model one is absurd - mildly said. The equal distribution of calories is an assumption from the utopist economics which is in contradiction to Malthusian framework. The following section of this paper will relax this assumption and explore the behavior of the Calorie model under weaker and more realistic assumptions of unequal distribution of calories and food market imperfections. Relaxing this assumption will help us to understand why there is death taking place before the stationary state itself is reached.

\section{Deaths and the stationary state}

This section will relax the assumption of equal distribution of calories among the population, but first it must explore the death rates in the stationary state. This is one aspect that is missing in the original article (Machay, 2012) because the author did not even consider the possibility of reaching the stationary state.

\section{Death rate in the Calorie model}

The stationary state is such a moment when the whole population can be sustained at the current level of technologies used in production for the last time. Formally, it can be written as

$c_{S}=\frac{A\left(t_{P}\right) a_{T}}{L\left(t_{P}\right) c_{N}}=1$.

Any time $t_{i}>t_{p}$ people will start dying because the means of production cannot sustain larger population. The maximum possible population that can be sustained can be expressed easily as
$L^{*}\left(t_{P}+1\right)=\frac{A\left(t_{P}+1\right) a_{T}}{c_{N}}$.

Because not all people can be sustained $\left(L\left(t_{p}+1\right)\right.$ $c_{N} \neq A\left(t_{p}+1\right) a_{T}$ formally) and since $g<n$ we can conclude that $L\left(t_{p}+1\right)>L^{*}\left(t_{p}+1\right)$. This leads to deaths occurring in the economy.

Now the only thing left to be done is to express how many people will actually die one time period after the stationary state took place.

$D\left(t_{P}+1\right)=L\left(t_{P}+1\right)-L^{*}\left(t_{P}+1\right)$

$D\left(t_{P}+1\right)=n L\left(t_{P}\right)-\frac{g A\left(t_{P}\right) a_{T}}{c_{N}}=n L\left(t_{P}\right)-g L\left(t_{P}\right)=(n-g) L\left(t_{P}\right)$

where $D\left(t_{p}+1\right)$ is amount of people who will die after the stationary state is reached. This result is intuitive because we clearly see that only those newly born will survive after the stationary state took place who can be sustained by additionally created calories as a consequence of the growth of technological level.

Expressing death rate in the Calorie model is as well simple. Using (5) and (6) for a fraction of those who were alive at the beginning of the given time period and those who can be sustained

$d_{P}=\frac{L\left(t_{P}+1\right)}{L^{*}\left(t_{P}+1\right)}=\frac{n L\left(t_{P}\right)}{\frac{g A\left(t_{P}\right) a_{T}}{c_{N}}}=\frac{n L\left(t_{P}\right)}{g L\left(t_{P}\right) c_{S}}=\frac{n}{g}$.

Death rate in the Calorie model as is shown in (8) is nothing else than positively dependent on the growth of population and negatively dependent on the growth of technological level. Based on (8) two new theorems are derived for the Calorie model that were not examined previously.

Theorem 1: The larger the growth of population the larger the death rate will be after the stationary state was reached by the economy.

Theorem 2: The larger the growth of technological level the smaller the death rate will be after the stationary state was reached by the economy.

The last question that still needs to be answered is what the growth of population will be after the stationary state will be reached. The solution is obvious. The growth of population will be lessened by the death rate. Moreover, using (8) we get that

$n_{P}=\frac{n}{d_{P}}=\frac{n}{\frac{n}{g}}=g$.

Theorem 3: After the stationary state is reached the growth of population will be equal to the growth of technological level.

This section added a new dimension to the Calorie model and derived the death rate to illustrate what it means to reach the stationary state. Next section will, however, examine deaths that precede the stationary state due to imperfections of reality. This is an aspect that was broadly considered within the Classical School framework. 


\section{Unequal distribution of calories}

If calories are distributed unequally it is a result of variation in the income each person disposes with - by Malthus labeled as 'the poor'. Hence, we can assume that there is a person whose income is different from the income each other person disposes with.

Assumption 1: Let us suppose there is equal distribution of income in the society except for one person whose income is $I_{N}=\alpha I_{0}$ for $\alpha>0$ and $\alpha \neq 1$.

This assumption replaces the assumption of equal distribution of calories in the society and from obvious reasons it is weaker than the original assumption of the model.

For this person has a different time of death than the others then must be true that in the time preceding her death all income will be spent for calories and the amount of calories purchased must be equal to $c_{N}$. Formally stated

$I_{N}=p(t) c_{N}$.

While for other members of the society must be true

$I_{0}=p_{X} X+p(t) c+p(t) c_{N}$ for $c>0$ and $p_{X} X \geq 0$

where $p_{X} X$ are expenditures of the consumer allocated to other goods than calories. From (10) and (11) we can conclude that $\alpha \in(0 ; 1)$ if the person is to die earlier than others. Now, substituting (4) in (10) and rearranging we get

$\gamma c_{N}=I_{N} e^{g t} a_{T}-I_{N} e^{n t} c_{N}$.

This is as much as the relaxed assumption will let us. Equation (12) has no solution without adding a new assumption because one cannot express time from (12). We can either set technological growth equal to zero or population growth to zero to get rid of the complication. From logical point of view it makes sense to set the technological growth to zero rather than the growth of population because doing so the society would actually move from the stationary state and not towards it. Notice the new assumption does not break the other assumption of the Calorie model nor the theorems derived from the model itself.

Assumption 2: Let us suppose the $g=0$.

Also notice that we replaced one very strong assumption with two weaker ones. However, both of them are still more realistic than the original one. Lets express time from (12) using assumption 2.

$$
\begin{aligned}
& \gamma c_{N}=I_{N} a_{T}-I_{N} e^{n t} c_{N} \\
& I_{N} e^{n t} c_{N}=I_{N} a_{T}-\gamma c_{N} \\
& e^{n t}=\frac{a_{T}}{c_{N}}-\frac{\gamma}{I_{N}}
\end{aligned}
$$

$t_{N}=\frac{\log \left(\frac{a_{T}}{c_{N}}-\frac{\gamma}{I_{N}}\right)}{n}$.

The last time period when this person will still be alive is seemingly expressed in (13) but there is a complication because due to $a_{T}$ and $\gamma$ being very large numbers one must prove that the expression in the logarithm is not negative for having an existing solution within the set of real numbers.

$\frac{a_{T}}{c_{N}}-\frac{\gamma}{I_{N}}>0$

$\frac{a_{T}}{c_{N}}>\frac{\gamma}{p(t) c_{N}}$

$a_{T}>\frac{\gamma}{\frac{\gamma}{e^{g t} a_{T}-e^{n t} c_{N}}}=a_{T}-e^{n t} c_{N}$.

The problem presented here has a solution in the set of real numbers because the expression in logarithm is positive as proved in (14). There is nothing in the way of confirming that the person will really die sooner than the others. This can be shown very easily

$t_{N}-t_{0}<0$

$\frac{\log \left(\frac{a_{T}}{c_{N}}-\frac{\gamma}{I_{N}}\right)}{n}-\frac{\log \left(\frac{a_{T}}{c_{N}}-\frac{\gamma}{I_{0}}\right)}{n}<0$

$\frac{a_{T}}{c_{N}}-\frac{\gamma}{\alpha I_{0}}<\frac{a_{T}}{c_{N}}-\frac{\gamma}{I_{0}}$

$1>\alpha$.

Which is true for $\alpha$. As a consequence of the model specification it is obvious that the lower the income $I_{N}$ is in relation to the income of others the sooner the person will die in relation to others. The model seems to be elegant because it yields intuitive results in compliance with Malthusian thoughts about the poor being the first ones to die. From relaxing the strong assumption we can get another theorem resulting from the model.

Theorem 4: The smaller the income of the person the sooner she will die even though the economy as a whole did not reach the stationary state yet.

This helps to theoretically explain what is going on in the world, especially in Africa, even though we still did not reach the global stationary state.

\section{Food market imperfections}

The original Calorie model does view the world as one market with free flow of calories. However, in reality we are far from a perfectly globalized 
economy. There are still significant trade barriers that prevent the free flow of food all around the globe. From this reason another death rate should be analyzed within the framework of the Calorie model. The one resulting from the unequal distribution of initial endowment of land that sustains the population. The globe shall be divided into two parts that cannot trade the calories while holding everything else constant.

Assumption 3: Let us suppose there are two societies on the planet which occupy two parts of it such as $a_{T 1}=\alpha a_{T}$ and $a_{T 2}=(1-\alpha) a_{T}$ and that the calories cannot flow from one part of the planet to the other one.

Each of the part of the globe dealing with individual conditions has unique calorie consumption surplus $c_{s 1}$ and $c_{s 2}$. These two societies have as a consequence two unique stationary states which can be expressed very easily as

$t_{P 1}=\frac{\log c_{N}-\log a_{T}-\log \alpha}{g-n}$ and

$t_{P 2}=\frac{\log c_{N}-\log a_{T}-\log (1-\alpha)}{g-n}$.

Let us further suppose that the stationary state in the first part of the world will come sooner than in the second one. For which $\alpha$ is this true?

$$
\begin{aligned}
& t_{P 1}-t_{P 2}<0 \\
& \frac{\log c_{N}-\log a_{T}-\log \alpha}{g-n}-\frac{\log c_{N}-\log a_{T}-\log (1-\alpha)}{g-n}<0 \\
& \frac{\log (1-\alpha)-\log \alpha}{g-n}<0 \\
& \log (1-\alpha)-\log \alpha>0
\end{aligned}
$$

$0.5>\alpha$.

Since the original theorem derived from the original Calorie model in the article (Machay, 2012) states that the rate of growth of population must be larger than the growth of technological level for the stationary state to occur it is easy to prove that for the stationary state to take place earlier in the first society alpha must be less then half of the available land for cultivation (as proved in 17). This is once again very intuitive and only proves elegance of the Calorie model and its conformity with the Classical School.

Theorem 5: If the planet is divided into two parts that cannot trade calories from whatever reason the stationary state will ceteris paribus take place in the less endowed society in advance to the more endowed society.

Calories are not the only thing that can be prevented from crossing the borders between our two societies. The technological transfer or technological diffusion can be prevented from several reasons. It can be anything from legal restrictions to bans to capital movement because it is the physical capital that is the carrier of technologies. Our next step will be to explore the variations in the growth of technological level.

Assumption 4: Let us suppose two identical societies sharing our planet for which only the growths of technological level are such as $g_{1} \neq g_{2}$ while other assumptions about them $\left(n>g_{i}>0\right.$ especially) remain unchanged.

The assumption 4 represents production imperfection in the form that the stationary state in one part could have been avoided in the case the technologies could move freely across the borderlines. Once again this will help us to make the Calorie model more realistic. Using similar construction as in (16) and assuming the stationary state will occur in the first society earlier we get

$$
\begin{aligned}
& \frac{\log c_{N}-\log a_{T}-\log \frac{1}{2}}{g_{1}-n}-\frac{\log c_{N}-\log a_{T}-\log \frac{1}{2}}{g_{2}-n}<0 \\
& \frac{1}{g_{1}-n}-\frac{1}{g_{2}-n}>0
\end{aligned}
$$

$g_{2}>g_{1}$.

We see in (19) that for the stationary state to occur in the society sooner the growth of technological level must be lesser than in the other society. This is again an intuitive conclusion that is formally stated in a following theorem.

Theorem 6: If there are two identical societies differing only in the growth of technological level the stationary state will occur sooner in the society with lower growth of technological level.

In a similar fashion we can assume different growths of population.

Assumption 5: Let us suppose two identical societies sharing our planet for which only the growths of population are such as $n_{1} \neq n_{2}$, while other assumptions about them $\left(n_{i}>g>0\right.$ especially) remain unchanged.

The proof is done in a similar way as the previous one. For the proof see the appendix. The following theorem is then a direct consequence of assumption 5 .

Theorem 7: If there are two identical societies differing only in the growth of population the stationary state will occur sooner in the society with larger growth of population.

There is one remaining variable that has not been explored in this article - the average amount of calories needed to sustain an average person alive. From obvious reasons this variable is not an object of economic inquiry. This short paper explored all possible variations in the death rates within the framework of the Calorie model and in the compliance with Malthus himself. 


\section{DISCUSSION}

This brief paper formalized and explored the stationary state and death rates of the Classical theory of population using the Calorie model which unlike the Neoclassical model of population is derived strictly within the Malthusian framework. All possible deaths were derived. The death rate was expressed because the original article (Machay, 2012) did not anticipate it as well as deaths resulting from imperfections and, thus, preceding the stationary state itself. This paper provided proofs of seven Malthusian theorems.

Moreover, the Malthusian system can be now easily formalized. First, let us speak Malthus for himself:

"During this season of distress, the discouragements to marriage, and the difficulty of rearing a family are so great, that population is at a stand. ... The situation of the labourer being then again tolerably comfortable, the restraints to population are in some degree loosened; and the same retrograde and progressive movements with respect to happiness are repeated." (Malthus, 1798)

The two movements of the society (pre-stationary and stationary) are easily expressible.

$m=\left\{\begin{array}{cl}n_{P} & \text { for } \quad c_{S} \rightarrow 1 \\ n & \text { otherwise }\end{array}\right.$

\section{REFERENCES}

BECKER, G. S., GLAESER, E. L. and MURPHY, K. M. 1999. Population and Economic Growth. The American Economic Review, 89(2): 145-149.

BIRCHENALL, J. A. 2015. Population and development redux. Journal of Population Economics, 29(2): 627-656.

GOLOSOV, M., JONES, L. E. and TERTILT, M. 2010. Efficiency with Endogenous Population Growth. Econometrica, 75(4): 1039-1071.

KREMER, M. 1993. Population Growth and Technological Change: One Million B.C. to 1990. The Quarterly Journal of Economics, 108(3): 681-716.

\section{Appendix A. Proof of Theorem 7}

Using assumption 5 and supposing that the stationary state will occur in the first society earlier than in the second one we can, in a similar fashion as (16) and (18), write

$$
\begin{aligned}
& \frac{\log c_{N}-\log a_{T}-\log \frac{1}{2}}{g-n_{1}}-\frac{\log c_{N}-\log a_{T}-\log \frac{1}{2}}{g-n_{2}}<0 \\
& \frac{1}{g-n_{1}}-\frac{1}{g-n_{2}}>0 \\
& n_{2}<n_{1}
\end{aligned}
$$

where $m$ is the movement of society. We clearly see that it is dependent on the consumption surplus. If it is close to one the society faces the retrograde movement and when it is very large the society enjoys a progressive movement.

But we are still far away from the final formalization of the Classical theory of population. The endogenous calorie price must be related to the situation in the labor market and as a consequence the incomes must be determined in a Classical fashion. The Calorie model should be estimated on the real $19^{\text {th }}$ century data. Further, the Classical model can be moved closer to the Neoclassicism by making the growth rates being time dependent but not interdependent. Also a detailed comparison between the Classical and Neoclassical models is missing in the literature. Formalization done in this paper should make this much easier. After all it seems there is much more in Malthus we still can use in modern era than a simple principle of population growing faster than the means of subsistence which is already a starting point of any population model.

MACHAY, M. 2012. The Principle of Population for the $21^{\text {st }}$ Century:TheNeverComingStationaryState. Review of Economic Perspectives - Národohospodárský obzor, 12(3): 168-184.

MADSEN, J. B., ANG, J. B. and BANERJEE, R. 2010. Four centuries of British economic growth: the roles of technology and population. Journal of Economic Growth, 15(4): 263-290.

MALTHUS, T. R. 1798. An Essay on the Principle of Population. $1^{\text {st }}$ Edition. London: J. Johnson, in St. Paul's Church-yard. 